\title{
Surfactant Excipient
}

National Cancer Institute

\section{Source}

National Cancer Institute. Surfactant Excipient. NCI Thesaurus. Code C42739.

A surface active agent (surfactant), in dilute solution, moves to the interface of a twophase system and reduces the surface free energy. This permits the two phases to mix more completely. Surface active agents are usually soluble both in aqueous and nonaqueous solvents. They are exemplified by soap-type molecules (long chain fatty acid salts) such as sodium lauryl sulfate. 